\title{
Norwood valved Sano shunt: Early reward versus late penalty?
}

\author{
Sameh M. Said, MD, and Joseph A. Dearani, MD
}

From the Department of Cardiovascular Surgery, Mayo Clinic, Rochester, Minn.

Disclosures: Authors have nothing to disclose with regard to commercial support.

Received for publication Dec 15, 2017; accepted for publication Dec 20, 2017; available ahead of print Jan 19, 2018.

Address for reprints: Joseph A. Dearani, MD, Department of Cardiovascular Surgery, Mayo Clinic, 200 First St SW, Rochester, MN 55905 (E-mail: jdearani@mayo.edu).

J Thorac Cardiovasc Surg 2018;155:1756-7

$0022-5223 / \$ 36.00$

Copyright (c) 2018 by The American Association for Thoracic Surgery

https://doi.org/10.1016/j.jtcvs.2017.12.114

There have been multiple modifications to the Norwood procedure over the last 2 decades, and the most important one has been the switch from the modified BlalockTaussig shunt to a right ventricular-to-pulmonary confluence conduit or "Sano conduit" (SC). The debate continues regarding the choice between the 2 , but more centers are in favor of using the $\mathrm{SC}^{1}$ because of improved hemodynamic stability in the early postoperative period.

Potential detriments of the SC include earlier cyanosis with the need for earlier second-stage palliation, potential systemic ventricular dysfunction, and dysrhythmias from ventriculotomy. ${ }^{2}$ In addition, the SC predisposes excess volume load on the single right ventricle from diastolic reversal of flow through the conduit. As a result, some authors proposed the use of a valved conduit to prevent the retrograde diastolic blood flow observed with the use of nonvalved conduits. Sandeep and colleagues ${ }^{3}$ reported their experience with 130 patients with hypoplastic left heart syndrome who underwent a modified stage I palliation with an SC conduit from 2002 to $2015 ; 100$ patients received a valved SC of a cryopreserved homograft valve anastomosed to a polytetrafluoroethylene tube, and 30 patients received the standard nonvalved SC. Although survival was better in the valved SC group $(P=.04)$, the difference was no longer significant after adjustment for the surgical era. Although there were more interventions for the valved SC group in the interstage period, it did not reach statistical significance $(P=.16)$, and there were no differences in right ventricular function at baseline or before stage II. The authors concluded that the valved SC was not associated with a survival benefit and did not confer an identifiable benefit on right ventricular function.

The strengths of this study include good early results of Norwood stage I and the largest reported number of patients who underwent the procedure with a valved SC. The main weakness includes the lack of detail of the potential benefits of the early postoperative physiologic and hemodynamic effects of the valved SC. The main advantage of the valved SC in the early postoperative period is the elimination of a

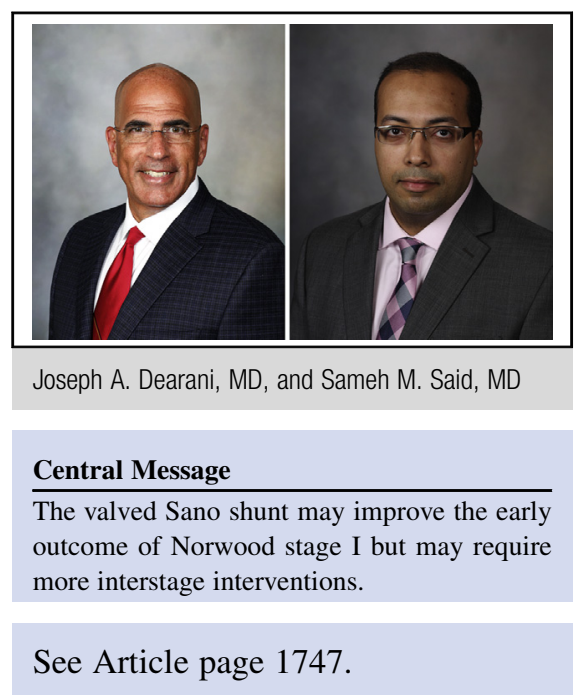

volume-loading lesion on the systemic ventricle that can contribute to more predictable hemodynamic stability. This may be particularly advantageous in less stable neonates undergoing the Norwood, those who required an atrial septostomy for restrictive atrial septal defect, or those with concomitant total anomalous pulmonary venous return. In addition, the more predictable stability of a valved SC in the early postoperative period may be beneficial in centers performing a smaller number of Norwood procedures. This advantage may not be apparent in a high-volume center such as the Stanford program, because their early outcomes are already good to excellent (early hospital mortality was not different between the valved and nonvalved groups; $P=.14$ ); thus, they focused on challenges and interventions of the SC during the interstage period.

The fundamental question is balancing the need and value of early hemodynamic stability versus the greater need of interstage interventions secondary to dysfunctional valves or conduits. It is clear that homograft valves may not last long enough to notice an actual benefit beyond the early postoperative period. This may be due to their smaller sizes, the pattern of failure (regurgitation vs stenosis), or whether it was an aortic or pulmonary allograft. Obstruction as a mode of failure is the more concerning mode of deterioration and will probably be associated with higher mortality because this is the only source of pulmonary blood; clearly, immediate intervention would be necessary. Detailed hemodynamic analysis of the early postoperative period was not performed to better understand what the cause of early 
deaths was and whether there was potential value of having a valve in the SC in patients with the risk factors outlined and whether there were differences between aortic and pulmonary homografts. Aortic homografts were used more frequently (vs pulmonary) in the valved group (51\% vs $35 \%$ ), and some studies have shown worse survival with the aortic versus pulmonary homografts, perhaps because of their greater susceptibility to obstruction, as opposed to regurgitation being the more common mode of deterioration in pulmonary homografts. Homograft availability likely plays a key role in these very small sizes. In the study by Sandeep and colleagues, ${ }^{3}$ all the patients with an intact atrial septum had a valved SC used, and this high-risk group probably benefitted from having a valve in their SC in the early postoperative period. Another consideration and potential advantage of a valved SC is when extracorporeal membrane oxygenation support may be necessary after the Norwood or if the child experienced cardiac arrest. The presence of a modified Blalock-Taussig shunt or nonvalved SC poses challenges (occlude vs leave open) while on extracorporeal membrane oxygenation support, and the presence of the valved SC may provide an additional hemodynamic benefit in this situation.

A valve in the SC may add some physiologic and hemodynamic benefit in the early postoperative period, particularly the higher-risk Norwood, but it comes with the disadvantage of a greater number of interventions during the interstage period. This "early reward" versus "late penalty" should be examined in more detail in a multiinstitutional study with a larger number of patients to examine this expanding role of a valve in the SC.

\section{References}

1. Pearl JM. Right ventricular-pulmonary artery connection in stage I palliation of hypoplastic left heart syndrome. J Thorac Cardiovasc Surg. 2003;126:1268-70.

2. Tanoue Y, Kado H, Shiokawa Y, Fusazaki N, Ishikawa S. Midterm ventricular performance after Norwood procedure with right ventricular-pulmonary artery conduit. Ann Thorac Surg. 2004;78:1965-71.

3. Sandeep N, Punn R, Balasubramanian S, Smith SN, Reinhartz O, Zhang Y, et al Interstage evaluation of homograft-valved right ventricle to pulmonary artery conduits for palliation of hypoplastic left heart syndrome. J Thorac Cardiovasc Surg. 2018;155:1742-55. 